\title{
Determinants of Perinatal Birth Asphyxia Among Preterm Babies in Ethiopia: A Systematic Review and Meta-Analysis of Observational Studies Protocol
}

\author{
Abay woday Tadesse ( $\square$ abaywoday@yahoo.com ) \\ Samara University, College of Medicine and Health Sciences, Afar Region, Ethiopia. \\ https://orcid.org/0000-0003-0499-4690 \\ Muluken Dessalegn \\ African Medical and Research Foundation
}

\section{Setognal Birara}

Samara University, Afar, Ethiopia.

\section{Kusse Urmale}

Samara University, Afar, Ethiopia

\section{Gebeyaw Biset}

Wollo University

Sisay Eshetie

Wollo UNiversity

Mekuriyaw Alemayehu

University of Gondar

\section{Protocol}

Keywords: Preterm babies, meta-analysis, birth asphyxia, difficulty of breathing at birth, associated factors, neonates, Ethiopia

Posted Date: May 27th, 2020

DOl: https://doi.org/10.21203/rs.3.rs-30470/v1

License: (c) (i) This work is licensed under a Creative Commons Attribution 4.0 International License. Read Full License 


\section{Abstract}

Background: Birth asphyxia among preterm babies accounted for nearly fifty percent of neonatal mortality in sub-Saharan African countries. This scenario has been worst in Ethiopia where every two out of three deaths attributed to birth asphyxia. Moreover, studies conducted in Ethiopia lack consistency in the prevalence and risk factors of birth asphyxia.

Objective: This study aims to analyses collectively and systematically prevalence of birth asphyxia and associated factors among preterm neonates in Ethiopia.

Methods: The protocol for this review is registered at PROSPERO with registration number CRD42020158224. A comprehensive search of the following electronic databases were made using: MEDLINE, EMBASE, CINAHL, Scopus, web science, HINARI, Cochrane library, Google Scholar and maternity \& infant care databases as well as grey literature uploaded at Ethiopian Health Development Journal will be searched until June 30,2020 . The quality of studies will be assessed using Joanna Briggs Institute (JBI) checklist. Three reviewers will screen all retrieved articles, conduct data extraction, and then critically appraise all identified studies. All identified observational studies reporting the prevalence of birth asphyxia and associated factors among neonates in Ethiopia will be considered. The analysis of data will be done using STATA 14.0 statistical software. We will demonstrate pooled estimates and determinants of neonatal mortality with effect size and $95 \%$ confidence interval. Heterogeneity among the included studies will be assessed through the Cochrane Q-test statistics and 12 test. Furthermore, publication bias will be checked using funnel plot and egger's test. Finally, statistical significance level will be declared at a p-value less than 0.05 .

Discussion: the result from this systematic review will inform and guide health policy planners to invest limited resources on maternal and neonatal health. Furthermore, it will be a stimulus for future cumulative meta-analysis researchers in developing nations.

\section{Background}

The World Health Organization (WHO) defines birth asphyxia as failure to initiate and sustain normal breathing at the first and fifth minute of birth [1]. Although more than two-thirds of newborns death are preventable, most of the neonatal deaths occur at home, uncounted, and invisible to national and regional policies and programs [2]. Globally, 2.5 million children and more than a million African babies are estimated to die in the first month of life, annually [3]. Worldwide, $25 \%$ of all neonatal deaths are attributed to birth asphyxia [4]. In Ethiopia, two-thirds of neonatal deaths are attributed to birth asphyxia [5]. Furthermore, birth asphyxia is the first cause of neonatal deaths (31.6\%) followed by prematurity $(21.8 \%)$ and sepsis (18.5\%) in developing countries, like, Ethiopia [6].

Studies conducted across the globe have identified factors associated with birth asphyxia. These include; antepartum risk factors (i.e. maternal age, maternal education, pre-eclampsia, primi-gravidity) intrapartum risk factors (i.e. prolonged labor, premature rupture of membrane, non-cephalic presentation, mode of 
delivery and maternal fever), and fetal risk factors (i.e. pre-term babies, fetal distress and baby weight) [7-11].

Globally and regionally various strategies and interventions have been implemented to decline neonatal mortality rates because of birth asphyxia $[3,4,12]$. For instance, one of the main objective in the Sustainable Development Goals of 2030 (SDG-3) is to reduce the neonatal mortality rate below 12 deaths per 1,000 live births [13]. Similarly, Ethiopia has made remarkable progress in achieving many of the national child health indicators [14] and it has designed the National Child Survival Strategy to reduce child mortality [15]. However, the prevalence of birth asphyxia varies across the globe and African countries contributes nearly fifty percent of the total magnitude of birth asphyxia [16-19]. Similarly, in Sub-Saharan countries, the prevalence of birth asphyxia is still unacceptably high which ranges from $21.3 \%$ to $56.9 \%$ [17, 20-24]. In Ethiopia, the magnitude of birth asphyxia varies between $3.1 \%$ and $56.9 \%$ in Ethiopia [23-26]. Therefore, none of these studies have shown pooled estimates of birth asphyxia and its determinants. Thus, central aim of this systematic review is to have pooled estimate of birth asphyxia and its determinants at national level, Ethiopia.

\section{Objective}

\subsection{General objective}

To estimate the pooled prevalence and associated factors of birth asphyxia among preterm newborns in Ethiopia.

\subsection{Specific objectives:}

- To determine the pooled prevalence of birth asphyxia among preterm newborns in Ethiopia

- To identify factors associated with birth asphyxia among preterm newborns

\section{Research Questions}

- What is the estimated prevalence of birth asphyxia among preterm babies in Ethiopia?

- What are the risk factors of birth asphyxia?

\section{Methods And Materials}

\subsection{Reporting of the review findings}

The protocol for this review is registered at PROSPERO with registration number CRD42020158224. We will use the Preferred Reporting Items for Systematic review and Meta-analyses (PRISMA-2009) [27] (additional file 3) and (PRISMA-P 2015) [28] (Additional file 1) statements to report the findings. Moreover, we have used the guideline of the Prospero for registration. 


\subsection{Study design}

A systematic review and meta-analysis will be conducted to determine the pooled prevalence of birth asphyxia and its determinants among newborns in Ethiopia.

\subsection{Eligibility Criteria}

\subsubsection{Inclusion criteria:}

Study Setting: This review will include studies conducted in Ethiopia.

Study Population: the review include only studies involving newborns with.

Study design: All observational studies (i.e. cross-sectional, case-control, and cohort) those have reported on prevalence of birth asphyxia and its determinants and have been published in peer-reviewed journals.

Language: studies reported in the English language will be considered.

Publication year: no restriction on year of publication

\subsubsection{Exclusion criteria:}

We will exclude studies published in languages other than English. We will exclude studies that include perinatal birth asphyxia. Additionally, we will exclude studies other than observational studies. case reports, conference reports, national survey reports and expert opinions.

\subsection{PECO search guide}

Population: Live newborns

Exposure: Predictors/determinants of birth asphyxia. The determinants are characteristics or exposures that increase the likelihood of birth asphyxia. These may be related to maternal residence, maternal age, educational status, newborn weight, and antenatal exposure.

Comparison: The reported reference group for each predictor/determinant in each study (e.g. birth asphyxia in mother living in urban area versus mothers living in rural area).

Outcome: We will include studies that assess perinatal birth asphyxia, if the studies have excluded stillbirth from perinatal mortality. 


\section{Searching strategy}

This meta-analysis will be prepared and presented according to the Preferred Reporting Items for Systematic Reviews and Meta-Analysis [PRISMA, 2009]. We will develop an appropriate and comprehensive search strategy with relevant search terms and pilot test it before the final search. We will search MEDLINE, EMBASE, CINAHL, Scopus, Web science, HINARI, Google Scholar, and Cochrane electronic Databases. We will include articles published from start of indexing until May 30, 2020. We will use Medical Subject Heading (Mesh), keywords, and free text search terms. As the search terms, we will include alternative terms for neonatal mortality, and will combine them using Boolean operators.

Search terms (Additional file 2\&3):: ((birth asphyxia OR "perinatal asphyxia" OR breathing difficulty at birth" OR low APGAR score) AND (determinants OR predictors OR "risk factors" OR causes) AND (newborn OR perinatal OR Neonate) AND (Ethiopia OR Ethio OR Etopia)

Moreover, we will utilize snowballing to screen the references of identified articles for potentially relevant studies. Furthermore, Studies identified by our database searching strategy will be retrieved and managed using Endnote X8 (Thomson Reuters, Philadelphia, PA, USA) software.

\subsection{Outcome measurement}

This systematic review and meta-analysis will have two main outcomes. The first outcome is estimating the pooled prevalence of birth asphyxia among the neonates. Birth asphyxia is defined as newborn with APGAR score less than 7 at the $1^{\text {st }}$ and $5^{\text {th }}$ minutes of birth. Pooled prevalence will be calculated by dividing the number of neonates with birth asphyxia to the total number of neonates who have been included in the study (total sample size) multiplied by 100 . The second outcome of the study is to identify maternal and neonatal factors associated with birth asphyxia. The predictors will be determined in the form of the log odds ratio.

\section{6 selection of studies}

Two authors (AWT, SB and GB) will review the studies, based on inclusion and exclusion criteria. The review will follow three stages. In the first stage, reviewers will assess the titles of the studies identified from the search. In the second stage, abstracts of these selected titles will be included for the final stage of full-text screening. In the third stage, full-text screening, we will screen the full texts selected in the previous stage. If the articles are not open access, we will contact the corresponding author at least for three times. If the authors are not willing to provide the full text, we will exclude that specific article.

In the review, we will only include those studies approved by both authors. The authors will resolve disagreements through discussion or consultation with a third reviewer $(\mathrm{KU})$. We will provide reason for exclusion for all excluded studies. Finally, we will prepare a final list of articles for data extraction 


\subsection{Data extraction}

Three authors (AW, SE and GB) will independently extracted all necessary data using a standardized data extraction format, which is adapted from the JBI data extraction format for observational studies [29]. We will pretest the data extraction form on three studies of each type, to ensure that it adequately facilitates the collection of all necessary data required for an effective systematic review and meta-analysis. Discrepancies between data extractors will be discussed to reach consensus. If a consensus cannot be reached, the authors will consult a third reviewer $(\mathrm{KU})$. For each included articles, we will record the first author's last name, year of publication, the setting where the study was conducted, study design, study period, sample size, the response rate, the population, outcome definition, comparison groups, and the effect estimate.

\subsection{Quality assessments}

Four authors (AWT, SB, KU and GB) will independently conduct quality assessment of included studies, by using the checklist of the JBI appraisal tool for cross-sectional, cohort and case-control studies (Additional file 3).. In customising the scale to fit this study, we will took into account the study sampling methods and similarities between the study groups regarding adjustment for confounding factors, the ascertainment of exposure and outcomes, and study design. The abstracting tool will include different questions based on the study designs. The four investigators independently will perform the quality assessment while abstracting the data for the meta-analysis. The quality scores of the four abstractors will be averaged. Finally, the studies with higher scores $(>50 \%)$ will be included into meta-analysis.

\subsection{Data synthesis and analysis}

The extracted data will be entered into a Microsoft Excel Database and then imported into STATA version 14.0 (Stata Corp LLC, Texas, USA) software with packages of Meta-analysis for further analysis. The researchers will perform a narrative description of the study population, the studies included, the risk factors identified, and the cause for birth asphyxia as well as the outcome characteristics. We will use tables and figures to summarize the selected studies and results.

The pooled prevalence of birth asphyxia in Ethiopia will be demonstrated. We will use fixed effect model if the studies have similar methodology, same population, and study design. If not, we will utilize the random effect model [30]. Then, the "metaprop" command with random-effects models at 95\% confidence intervals will be used to calculate the pooled prevalence of birth asphyxia at national level [31]. The Freeman Tuckey variant of the arcsine square root transformation of proportions will be fitted to avoid variance instability when handling proportions close to one [32]. We will assess heterogeneity by using chi-squared test on Cochran's Q statistic with a $5 \%$ level of statistical significance [26] and $\mathrm{I}^{2}$ statistic test [33], assuming that $\mathrm{I}^{2}$ value of $25 \%, 50 \%$, and $75 \%$ being representative of low, moderate, and 
high heterogeneity, respectively [33]. If the heterogeneity is significant $\left(1^{2}>75 \%\right)$, then, we will conduct subgroup analyses and meta-regression to investigate sources of heterogeneity.

Publication bias will be examined by the visual inspection of funnel plots [34] and Egger's test [35]. A p value $<0.10$ will be considered indicative of statistically significant publication bias. Thus, if there is evidence of publication bias, we will use Duval and Tweedie's trim-and-fill method [36].

For factors associated with birth asphyxia; two-by-two tables will be constructed (if possible), the odds ratio with $95 \%$ confidence interval will be calculated. Then, the statistically significance level will be declared at a p-value less than 0.05 . However, if the meta-analysis is not possible, we will conduct narrative synthesis.

\subsection{Subgroup and sensitivity analyses}

Sub-group analysis will be performed based on study design, sample size, regions or state, year of publication, quality of studies, and study settings of included studies. by years of publication, study setting, study design, and sample size of the studies. Finally, to conduct sensitivity analysis, we will assess the stability or robustness of the pooled estimates to outliers and the impact of individual studies [36].

\subsection{Discussion}

This review will provide a detailed summary of the evidence on prevalence and factors of birth asphyxia in Ethiopia. This review will be the first to synthesis available data on birth asphyxia. Findings of the review will fill an evidence gap in understanding the burden, risk factors, and causes of birth asphyxia in the country. The result from this review will inform health policy planners and researchers up-to-date data on birth asphyxia and provide direction in which risk factors the policy should focus to reduce burden of birth asphyxia in the country.

\subsection{Dissemination of results}

The results of this systematic review and meta-analysis will be published in a peer-reviewed journal and presented at national and international research conferences.

\section{Abbreviation}

EDHS Ethiopian Demographic and Health Survey.

JBI Joanna Briggs Institute.

LMICs $\quad$ Low and Middle Income Countries. 
MeSH Medical Subject Headings.

PECO Population, Exposure, Comparison, and Outcome.

PRISMA Preferred Reporting Items for Systematic Review and Meta-Analysis.

WHO World Health Organization.

\section{Declarations}

\section{Ethics approval and consent to participate}

Not applicable

\section{Availability of data and materials}

All materials and data related to this article are included in the main document of the manuscript. However, if anyone has interested to have raw data, he/she can contact the corresponding author.

\section{Consent for publication}

Not applicable

\section{Competing interests}

The authors have declared that there are no competing interests.

\section{Funding}

No funding was used.

\section{Acknowledgements}

We would like to thank Samara university librarian and ICT complex staffs for availing an uninterrupted internet connection. We also acknowledge PROSPERO for registration of this protocol.

\section{References}

1. WHO. RECOMMENDED DEFINITIONS, TERMINOLOGY AND FORMAT FOR STATISTICAL TABLES RELATED TO THE PERINATAL PERIOD AND USE OF A NEW CERTIFICATE FOR CAUSE OF PERINATAL 
DEATHS: Modifications Recommended by FIGO as Amended October 14,1976:. Acta Dbstet Oynecoi Scand: 1977, 56:247-253.

2. Joy Lawn PM, Simon Cousens. Africa's newborns- counting them and making them count. Opportunities for Africa's Newborns 2010.

3. Newborns: reducing mortality [https://www.who.int/news-room/fact-sheets/detail/newbornsreducing-mortality]

4. WHO. GUIDELINE ON BASIC NEWBORN RESUSCITATION. Geneva 27, Switzerland 2012.

5. Hayelom Gebrekirstos Mengesha aBWS: Cause of neonatal deaths in Northern Ethiopia: a prospective cohort study. BMC Public Health 2017, 17(62):1-8.

6. UNICEF FEC Maternal and Newborn Health Disparities in Ethiopia. COUNTRY PROFIE_ETH 2015.

7. Aslam HM, Saleem S, Afzal R, lqbal U, Saleem SM, Shaikh MW, Shahid N. "Risk factors of birth asphyxia". Ital J Pediatr 2014, 40:94.

8. Farhana Tabassum AR, Shabina Ariff, Sajid Soofi, Zulfiqar A. Bhutta. Risk Factors Associated with Birth Asphyxia in Rural District Matiari, Pakistan: A Case Control Study. International Journal of Clinical Medicine 2014, 5:1430-1441.

9. Anália Luana Sena de Souza NLdS, Débora Feitosa de França, Samara Isabela Maia de Oliveira, Anne Karoline Candido Araújo, Dândara Nayara Azevedo Dantas. Risk Factors for Perinatal Asphyxia in Newborns Delivered at Term. Open Journal of Nursing, 2016, 6:558-564.

10. Tasew H, Zemicheal M, Teklay G, Mariye T, Ayele E. Risk factors of birth asphyxia among newborns in public hospitals of Central Zone, Tigray, Ethiopia 2018. BMC Res Notes 2018, 11(1):496.

11. Chiabi A, Nguefack S, Mah E, Nodem S, Mbuagbaw L, Mbonda E, et al. Risk factors for birth asphyxia in an urban health facility in cameroon. Iranian journal of child neurology 2013, 7(3):46-54.

12. WHO. WHO recommendations on interventions to improve preterm birth outcome. France; 2015.

13. UN. Transforming our world: the 2030 Agenda for Sustainable Development. $h t t p: / / w w w u n o r g$ /sustainable development/health/2015.

14. FMoFED [Ethiopia] Assessing progress towards the millenium development goals: Ethiopia MDGs report 2012. In. Addis Ababa, Ethiopia; 2012: 42.

15. FMOH [Ethiopia]. National Newborn and Child Survival Strategy Document Brief Summary 2015/162019/20:. In. Addis Ababa; 2015.

16. Halloran DR, McClure E, Chakraborty H, Chomba E, Wright LL, Carlo WA: Birth asphyxia survivors in a developing country. J Perinatol 2009, 29(3):243-249.

17. Ibrahim Aliyu TOL, Ben Onankpa.: Prevalence and outcome of perinatal asphyxia: Our experience in a semi-urban setting. Trop J Med Res 2017, 20:161-165.

18. Ilah B, Aminu M, Musa A, Adelakun M, Adeniji A, Kolawole T: Prevalence and Risk Factors for Perinatal Asphyxia as Seen at a Specialist Hospital in Gusau, Nigeria. Sub-Saharan African Journal of Medicine 2015, 2(2):64. 
19. Mukhtar-Yola M, Audu LI, Olaniyan O, Akinbi HT, Dawodu A, Donovan EF: Decreasing birth asphyxia: utility of statistical process control in a low-resource setting. BMJ Open Qual 2018, 7(3):e000231.

20. Ersdal HL, Mduma E, Svensen E, Perlman J: Birth asphyxia: a major cause of early neonatal mortality in a Tanzanian rural hospital. Pediatrics 2012, 129(5):e1238-1243.

21. Evelyn Mah-Mungyeh AC, Fanny Lorraine Tchokoteu, Seraphin Nguefack, Jean Baptiste Bogne Hyppolyte Siyou H, Florence Soh Fru , Jacob Enoh, Elie Mbonda , Pierre Fernand Tchokoteu.: Neonatal mortality in a referral hospital in Cameroon over a seven year period: trends, associated factors and causes. African Health Sciences: 2014, 14(4).

22. Gdiom Gebreheat TT, Dessalegn Kiros, Hirut Teame, Natnael Etsay, GueshWelu, and Desta Abraha.: Prevalence and Associated Factors of Perinatal Asphyxia among Neonates in General Hospitals of Tigray, Ethiopia. BioMed Research International 2018.

23. Solayman M HS, Akber T , Islam MI, Islam MA.: Prevalence of Perinatal Asphyxia with Evaluation of Associated Risk Factors in a Rural Tertiary Level Hospital. KYAMC Journal 2017, 8(1).

24. Woday A, Muluneh A, St Denis C: Birth asphyxia and its associated factors among newborns in public hospital, northeast Amhara, Ethiopia. PLoS One 2019, 14(12):e0226891.

25. DH. RAaD: Morbidities, Rate and Time Trends of Neonatal Mortality in Dilchora Referral Hospital, Dire Dawa, Ethiopia, 2012-2017 Austin Med Sci 2017, 2(1).

26. Neil Abdurashid Ibrahim AMaSA: Prevalence of Birth Asphyxia and Associated Factors among Neonates Delivered in Dilchora Referral Hospital, in Dire Dawa, Eastern Ethiopia. Clinics Mother Child Health 2017, 14(4).

27. Moher D, Liberati A, Tetzlaff J, Altman DG: Preferred reporting items for systematic reviews and meta-analyses: the PRISMA statement. Bmj 2009, 339:b2535.

28. Shamseer L, Moher D, Clarke M, Ghersi D, Liberati A, Petticrew M, Shekelle P, Stewart LA: Preferred reporting items for systematic review and meta-analysis protocols (PRISMA-P) 2015: elaboration and explanation. BMJ : British Medical Journal 2015, 349:g7647.

29. Institute JB: Joanna Briggs Institute Reviewers Manual 2014 Edition. In., 2014 edn. Australia: the University of Adelaide; 2014

30. DerSimonian R, Laird N: Meta-analysis in clinical trials. Controlled clinical trials 1986, 7(3):177-188.

31. Berkey CS, Hoaglin DC, Mosteller F, Colditz GA: A random-effects regression model for meta-analysis. Stat Med 1995, 14(4):395-411.

32. NN. V, A. M, A. M: Metaprop: a Stata command to perform meta-analysis of binomial data. Archives ofPublic Health 2014, 72(39).

33. Higgins JP, Thompson SG: Quantifying heterogeneity in a meta-analysis. Stat Med 2002, 21(11):1539-1558.

34. Liu JL: The role of the funnel plot in detecting publication and related biases in meta-analysis. Evidence-based dentistry 2011, 12(4):121-122. 
35. Egger M, Davey Smith G, Schneider M, Minder C: Bias in meta-analysis detected by a simple, graphical test. Bmj 1997, 315(7109):629-634.

36. Duval S, Tweedie R: Trim and fill: A simple funnel-plot-based method of testing and adjusting for publication bias in meta-analysis. Biometrics 2000, 56(2):455-463. 